\title{
Introduction. L'incomplète mise en politique du sous-sol français
}

\author{
Xavier Arnauld de Sartre ${ }^{1, *}$ (D) et Sébastien Chailleux ${ }^{2}$ (D) \\ ${ }^{1}$ Géographie, Centre national de la recherche scientifique / Université de Pau et des Pays de l'Adour, E2S UPPA, \\ UMR TREE, ICL, Pau, France \\ 2 Sciences politiques, Université de Pau et des Pays de l'Adour, E2S UPPA, UMR TREE, ICL, Pau, France
}

\begin{abstract}
Résumé - L'introduction de ce numéro spécial sur la politisation du sous-sol se propose d'analyser les utilisations du sous-sol qui émergent du fait des changements globaux et les conflits autour de ces utilisations, au travers du prisme de la politisation d'un milieu. Il part pour cela de la spécificité de ce milieu, pour lequel aucune expérience directe n'est possible et dont la connaissance et l'utilisation passent donc par le truchement de techniques. Cette caractéristique explique pour partie les formes particulières que prennent les contestations des usages du sous-sol, qui oscillent entre enrôlements des populations, contestations éruptives et critiques radicales du capitalisme. Or, les utilisations du sous-sol et leurs contestations ne conduisent pas nécessairement à une mise en politique, c'est-à-dire une mise en choix d'une option technique en matière de transition énergétique. Soumettant les articles du numéro spécial à une grille de lecture de la politisation qui implique que les utilisations soient crédibles, gouvernables et légitimes, cette introduction montre l'incomplétude de la politisation du sous-sol français.
\end{abstract}

Mots-clés : politisation / sous-sol / transition énergétique / géologie politique / France

Abstract - The incomplete politicization of France's subsurface. The introduction to this special issue on the politicization of the subsurface aims to analyse the multiple uses of the subsurface that are emerging due to global changes, and the conflicts about these uses, through the prism of the politicization of this environment. The paper starts from the specific nature of this environment for which no direct experience is possible and whose knowledge and utilization must therefore depend on techniques. This partly explains the particular forms taken by opposition to the uses of the subsurface, which range from population enlistment, eruptive social movements to radical criticism of capitalism. However, the uses of the subsurface and their contestation do not always lead to politicization, meaning that no public debate takes place about the choice of a technical option in terms of energy transition. Reading the articles in this special issue from the analytical standpoint of politicization, which implies that uses should be credible, governable and legitimate, this introduction shows the insufficient politicization of French subsurface.

Keywords: politicisation / energy transition / subsurface / political geology / France

Ce numéro spécial part du constat suivant: les soussols sont aujourd'hui au centre d'enjeux sociaux, environnementaux, économiques et politiques cruciaux pour nos sociétés, que ce soit du fait des contraintes posées par les changements climatiques aux ressources énergétiques fossiles (hydrocarbures), des contestations de projets d'extraction de ressources minérales («renouveau minier ») ou de nouvelles énergies (géothermie profonde, hydrogène natif), de la mise en débat des

\footnotetext{
*Auteur correspondant : xavier.arnauld@cnrs.fr
}

projets de stockage souterrain de ressources (gaz naturel, hydrogène) ou de déchets (nucléaires, chimiques ou bien encore $\mathrm{CO}_{2}$ ) et bien plus largement encore de la réforme du Code minier.

La thèse que nous défendons dans ce numéro spécial est qu'en dépit de cette actualité prégnante, le sous-sol reste largement un impensé de la transition énergétique française : il ne fait l'objet ni de représentations unifiées ni de débats publics qui permettraient sa mise en politique au service, notamment, de la transition énergétique. Ce n'est pourtant pas faute d'être contesté : 
la liste des contestations de l'utilisation du sous-sol est longue et montre que celui-ci est largement enrôlé dans des combats fortement politisés. Mais s'il est saisi comme un problème donnant lieu à des échanges d'argumentaires entre opposants et porteurs de projets, ces débats ne sont que rarement encadrés et il ne fait pas l'objet d'un arbitrage et d'un portage politique, ce qui permettrait d'en faire un objet politique unifié au service d'une action publique.

Nous nous proposons, dans cette introduction, de décrire le processus qui permet de considérer que le soussol revient dans les débats contemporains sans pour autant donner lieu à une véritable stratégie politique de son utilisation dans la transition énergétique. Pour cela, nous commençons par décrire les différentes utilisations du sous-sol et leurs contestations. Nous décrivons en particulier les formes que prennent ces mobilisations et la manière dont elles participent à des luttes plus larges. Nous expliquons cependant dans une seconde partie pourquoi cet enrôlement du sous-sol dans des luttes politiques ne saurait être qualifié de politisation de ce milieu. Développant un cadre théorique s'inspirant des critiques de la technique comme solution aux changements globaux, nous montrons comment les articles du numéro spécial ne permettent pas de lire, dans les réponses qui sont apportées aux contestations, une politisation du sous-sol.

\section{Renouveau du sous-sol et de ses contestations}

\section{Le sous-sol, un milieu incertain et confiné}

Pour comprendre les formes que prend la réapparition du sous-sol dans le débat public, il importe de commencer par en rappeler une spécificité forte : son expérience directe par les sens est impossible et les savoirs le concernant passent par le truchement de savoirs experts extrêmement coûteux, et le plus souvent aux mains de groupes industriels chargés de son exploitation.

Les savoirs du sous-sol sont très spécialisés et une médiation technique est nécessaire pour y accéder. Contrairement aux autres aspects de notre environnement plus ou moins directement perceptibles, le monde souterrain n'est pas sensible et les spécialistes, géologues et ingénieurs, ont recours à toute une instrumentation pour émettre des hypothèses sur la structure du sous-sol : études sismiques, forages exploratoires, carottages, etc. Ces savoirs experts sont produits en étroite collaboration avec l'industrie, qu'elle soit minière, pétrolière ou gazière. Les coûts de l'exploration géologique sont en effet tels qu'aucun organisme public n'a les moyens d'engager des forages qui auraient la connaissance pour seul objectif. C'est d'ailleurs dans un esprit de mutualisation des données du sous-sol qu'est organisé le Code minier français, qui ne prévoit pas de redevances très élevées, mais qui, en revanche, prévoit que les compagnies divulguent leurs données d'exploration à l'administration afin que celle-ci dispose d'une banque de données géologiques. C'est sur une sorte de partenariat public/privé que repose la connaissance du sous-sol.

Or, celui-ci peut poser problème, pour deux raisons. D'abord parce qu'il existe de véritables lacunes dans la connaissance du sous-sol: le sous-équipement des Directions régionales de l'environnement, de l'aménagement et du logement (Dreal) a notamment été soulevé durant la controverse sur le gaz de schiste en 2011, puisque, face à la recrudescence des demandes de permis dans des zones jusque-là sans industrie d'exploration pétrogazière, certaines Dreal étaient démunies en termes d'expertise des demandes. En outre, même dans des zones explorées, le degré d'incertitude reste très élevé : la connaissance du sous-sol est très imparfaite et on peut très difficilement inférer d'un carottage ou des enregistrements permis par un camion vibreur un savoir généralisable sur une zone. Cela rend l'exploitation du sous-sol particulièrement aléatoire et les savoirs le concernant incertains. Or, quand une incertitude ne peut être levée que par le truchement de savoirs acquis et détenus par les acteurs qui ont intérêt à exploiter le milieu, il va de soi que la controverse part sur de mauvaises bases - d'autant que toute contre-expertise est particulièrement compliquée et coûteuse (Wylie, 2018).

\section{Nouveaux usages du sous-sol}

Le sous-sol a fourni les ressources fondamentales au déploiement de la modernité : c'est de ce milieu qu'ont été extraits les matières premières, matériaux et énergies, qui ont permis que se développent les trois révolutions industrielles (Huber, 2009; Pomeranz, 2010 ; Bonneuil et Fressoz, 2013; Moore, 2017). L'exploitation des soussols a ainsi eu un impact très puissant sur l'organisation des sociétés en surface (Clark, 2016; Mumford, 2016). De ce fait, le sous-sol a fait l'objet d'une attention particulière au cours $d u \mathrm{XIX}^{\mathrm{e}}$ siècle et au début du $\mathrm{Xx}^{\mathrm{e}}$ siècle, visible notamment dans le droit minier (Braun, 2000), la répartition des richesses et le contrôle des travailleurs du sous-sol (Mitchell, 2013). Mais ce lien s'est progressivement distendu en Europe (Chailleux, 2020) : alors que le passage du charbon au pétrole a eu pour effet d'accroître encore la dépendance au sous-sol, il a permis un découplage entre les sociétés et leurs soussols. En effet, en délocalisant la production charbonnière de l'Europe vers les champs pétroliers moyen-orientaux, en permettant de découpler la production de l'énergie de son utilisation et en réduisant le besoin de main-d'œuvre que nécessitait l'exploitation du charbon, la transition du 
charbon vers le pétrole a éloigné les populations occidentales du sous-sol (Mitchell, 2013) et a d'autant plus facilement fait disparaître les débats publics que les mines permettant d'extraire des minerais ont elles aussi peu à peu fermé, réorientant l'action publique vers les problématiques d'après-mine (Chailleux et al., 2018). Ainsi, s'il reste encore quelques concessions minières (principalement de sel) et pétrolières (bassins parisien et aquitain) en France métropolitaine, ce sont deux industries marginales depuis la fin des années 1990.

Or les changements globaux, et notamment les changements climatiques, sont en train de changer cette situation: les sous-sols sont aujourd'hui au centre de nouveaux enjeux, que ce soit par la négative (l'opposition aux extractions du sous-sol) ou par la mobilisation du soussol pour la transition énergétique. Le sous-sol est en effet à la fois le lieu d'où sont extraits les composés chimiques qui génèrent les changements climatiques, mais aussi un milieu utile pour certaines formes de transition énergétique : il a connu un renouveau ces dernières années pour l'extraction de ressources minérales utiles à la transition énergétique (tungstène, cobalt, néodyme indispensables pour les batteries des voitures électriques et les aimants des éoliennes), pour le développement d'énergies dites de transition (gaz de schiste ou de houille défini comme bridging fuel), pour la création de nouvelles énergies (chaleur par géothermie profonde ou utilisation d'hydrogène natif), pour le stockage d'énergie (gaz naturel et, de manière croissante, hydrogène) ou de déchets (nucléaires, chimiques ou bien encore $\mathrm{CO}_{2}$ ). Or ce regain d'intérêt se traduit par l'émergence de nouvelles contestations, le soussol devenant un symbole des débats autour de la transition énergétique.

\section{Trois formes de contestation des utilisations industrielles du sous-sol}

Le sous-sol est contesté non seulement pour sa contribution aux changements climatiques (au travers de l'exploitation de combustibles fossiles), mais aussi à travers sa mobilisation comme solution contre les changements climatiques (des oppositions aux projets de stockage géologiques jusqu'à celles contre le renouveau minier en passant par la géothermie). Ces oppositions ne sont pas nouvelles, mais elles sont à la fois plus visibles et plus globales. En effet, les historiens de l'environnement ont montré que les oppositions aux aménagements, aux technologies et à leurs impacts sociaux et/ou environnementaux sont aussi anciennes que les projets qui les accompagnent (Fressoz, 2012; Bonneuil et Fressoz, 2013; Pessis et al., 2013). Ces contestations ont toutefois gagné en visibilité du fait de la montée en puissance des changements globaux dans les agendas médiatiques et politiques, générant de nouveaux types de problématisation du sous-sol. Elles sont aussi devenues plus globales à travers la coalition de trois types d'opposition.

Le premier type d'opposition est limité à l'action des riverains. Les techniques d'ingénierie du sous-sol sont connues pour provoquer des effets localisés non intentionnels. Ce sont principalement des fuites de fluides et de gaz, qui peuvent remonter et circuler dans les milieux de surface, ou la sismicité induite par les activités industrielles. En outre, ces activités génèrent des activités en surface (usines de traitement, transports) qui vont avoir des impacts sur les riverains. Ces effets locaux produisent ainsi des contestations qui sont généralement traitées par la création de normes et le paiement d'indemnités aux riverains (Fressoz, 2012).

Le second type d'opposition est fondé sur des arguments écologiques. Le principal argument aujourd'hui est bien entendu celui lié au rejet de gaz à effet de serre, confinés dans le sous-sol depuis des temps géologiques et libérés soit par combustion, soit directement dans l'atmosphère, provoquant les changements climatiques. D'autres arguments de nature écologique émergent également: pollutions liées à l'utilisation de produits chimiques pour la purification des produits issus du sous-sol (cyanure, mercure, etc.), stockage des résidus, impacts sur la biodiversité, perturbation des cycles biochimiques, etc.

Enfin, le troisième type d'opposition, plus politique, s'oppose au «système» que l'exploitation du sous-sol permet de maintenir et cible la structure capitaliste comme responsable des maux sociaux, politiques, économiques et environnementaux découlant de l'exploitation industrielle du sous-sol. L'analyse des oppositions à l'exploitation des hydrocarbures qui s'est manifestée à la COP 21 a montré que les oppositions ne portaient pas seulement sur les hydrocarbures, mais sur le «monde» véhiculé par les énergies carbonnées, à savoir un monde «technoscientifique», que les contestataires opposent à un monde socio-écologique (Aykut et CastroLarrañaga, 2017).

Ces trois logiques de contestations sont, sur le terrain, difficilement distinguables - elles sont au contraire profondément imbriquées, mais leur segmentation par les porteurs de projet, et aussi par les services de l'État, donne lieu à des réponses souvent inefficaces et contribue à limiter la possibilité de délibération collective.

\section{Une problématisation située entre captation des publics, contestations environnementales et radicalisation des oppositions}

Les contestations des utilisations du sous-sol se manifestent de trois manières distinctes dans les débats sur la transition énergétique : par la captation des publics, les contestations environnementales et l'anti-extractivisme. 
La captation des publics dans des arènes particulières découle de l'application des procédures classiques de concertation. Ce processus n'est pas propre au sous-sol, mais le confinement des connaissances liées au monde souterrain rend le débat public profondément dépendant du partenariat public-privé mentionné. Le risque que les publics soient « captés » par les dispositifs de participation est connu (Gourgues et al., 2013): en effet, si ces dispositifs ouvrent des espaces d'implication réelle des publics, ils cadrent et canalisent les enjeux, les acteurs et les débats qui s'y tiennent (Barthe, 2006; Gourgues, 2013; Chailleux, 2016). Dans la mesure où le savoir sur le soussol est une affaire d'experts, les risques de captation des publics dans des arènes confinées sont décuplés dans le cas de technologies mobilisant le sous-sol. En France, les commissions de suivi de site, qui remplacent depuis 2012 les commissions locales d'information, représentent le principal outil réglementaire visant à intégrer les populations. Qu'il s'agisse d'une installation nucléaire, d'un centre de stockage de déchets ou encore d'une installation industrielle à risque technologique, la préfecture d'un territoire doit mettre en œuvre une telle arène qui rassemble l'État, les collectivités territoriales, l'exploitant, les salariés de l'exploitant et les représentants de la population, le plus souvent des associations.

Or, si leur objectif officiel est d'intégrer les acteurs pertinents du projet afin de produire « un lieu de débats et de consensus ${ }^{1} »$, leur fonctionnement ne protège pas contre la mise en place d'une asymétrie entre les parties prenantes. Asymétrie de savoirs d'abord : c'est l'exploitant qui fournit les informations. Asymétrie d'accès à la parole ensuite : ce sont les services de l'État qui constituent la liste des membres des commissions. Asymétrie de cadrage enfin : les ordres du jour et les grands enjeux sont posés encore une fois par le président de la commission - qui doit veiller néanmoins à ce que l'ordre du jour soit participatif. Le paradoxe maintes fois souligné des dispositifs de participation s'applique au soussol et fait courir le risque que les publics ne puissent s'exprimerque dans les cadres établis, relativement aveugles aux problèmes émergents et particulièrement victimes de « régime d'imperceptibilité ${ }^{2} »$ (Wylie, 2018).

\footnotetext{
${ }^{1}$ Ministère de l'Écologie, du Développement durable et de l'Énergie, 2012. Circulaire du 15 novembre 2012 relative à la mise en application du décret $n^{\circ}$ 2012-189 du 7 février 2012 relatif aux commissions de suivi de site 2012 .

${ }^{2}$ À la suite des travaux sur l'agnotologie et la production d'ignorance, la notion de régime d'imperceptibilité vise à décrire la structuration d'un domaine de savoir en fonction de ce qu'il passe sous silence. Wylie (2018) développe l'exemple de la nocivité des perturbateurs endocriniens que ni la structure des organismes régulateurs ni celle des instituts de recherche disciplinaire ne permettait de démontrer et elle souligne le rôle de l'industrie pétrochimique dans le maintien de l'incertitude, notamment en lien avec le déploiement de la fracturation hydraulique aux États-Unis.
}

De plus, ces commissions reproduisent un prisme localiste de la contestation qui ne serait qu'une affaire de riverains et contribue à renforcer la critique des porteurs de projet sur l'importation d'une problématique écologique par des membres extérieurs à la communauté concernée. Jusqu'à il y a peu, la littérature a ainsi beaucoup insisté sur le phénomène bien connu de Nimby (Trom, 1999) et sur l'importance fondamentale de la proximité spatiale dans la contestation des projets d'aménagement (Kirat et Torre, 2008). Or, on sait que cette catégorie a notamment été utilisée par les porteurs de projet pour décrédibiliser les opposants (Batellier, 2016; Boissonade et al., 2016). Et pourtant, si la littérature sur les nouvelles ressources des sous-sols mobilise encore parfois cette catégorie (Krause et al., 2014), les opposants ne sont en aucune manière attachés par un déterminisme à la vision du monde que leur confère leur environnement local. Si ces mécanismes de captation des publics parviennent dans certains cas à confiner les problématiques - et ainsi à limiter le débat - , ils réussissent rarement à empêcher une contestation moins encadrée.

Il est en effet tout aussi possible que des publics se constituent en dehors des cadres institutionnalisés et forment un mouvement social qui va essayer de mettre à l'agenda un problème public autour du projet d'utilisation du sous-sol. Ce mouvement social est à la fois ancré dans des réseaux locaux et globaux et, s'il dénonce des atteintes à l'environnement local, c'est sur la base d'une montée en généralité traduite dans le slogan «ni ici, ni ailleurs ». Les mouvements sociaux ciblant tout ou partie $\mathrm{du}$ sous-sol constituent une part importante des contestations écologiques de la dernière décennie. On peut en distinguer deux aspects souvent conjoints: la dimension de l'urgence environnementale et celle du modèle de développement.

La dénonciation d'impacts environnementaux est au cœur des contestations ciblant le sous-sol, qu'il s'agisse de mines, de forages pétroliers ou géothermiques. Si ces impacts peuvent avoir une portée localisée (pollutions de nappes phréatiques ou de cours d'eau), d'autres sont globaux (gaz à effet de serre). Les opposants réalisent le plus souvent une montée en généralité entre leur problème localisé et l'ampleur d'une catastrophe à venir, notamment à travers la multiplication des permis de recherche sur différentes zones qui permet la mise en réseaux de collectifs locaux formant de puissants relais auprès des élus locaux et nationaux. Lorsque les opposants au gaz de schiste ou aux mines adoptent le slogan «ni ici, ni ailleurs », leur argument est fondé sur le registre du refus d'un monde et non plus seulement d'un projet. Il faut toutefois distinguer la particularité des «nouvelles» utilisations du sous-sol en matière de critique environnementale, puisque les oppositions contre le stockage géologique de carbone ou la géothermie ne rencontrent pas de telle mise en absolu de la contestation. En effet, 
si la problématique environnementale demeure importante, elle reste limitée en termes de risques locaux.

Enfin, ces luttes sont à rapprocher du mouvement contre l'extractivisme (Gudynas, 2009; Bebbington et Bury, 2013 ; Bednick, 2015), qui incarne la radicalisation des oppositions à l'exploitation du sous-sol et la dénonciation d'un modèle de développement fondé sur l'extraction des ressources du sous-sol. Le mouvement anti-extractivisme émerge en Amérique latine dans les années 1980 et fait l'objet d'un compte rendu de lecture croisé dans cet ouvrage. Si l'extractivisme ne concerne pas uniquement le sous-sol, il permet de souligner un régime de dépossession et d'accumulation des richesses commun à un ensemble d'activités dont en première ligne les mines et les hydrocarbures. Ce régime est aussi renforcé par le progrès technologique qui ouvre de nouveaux territoires à l'extraction comme la fracturation hydraulique (Svampa, 2016). L'extractivisme est longuement critiqué pour les inégalités qu'il génère, son caractère antidémocratique et les transformations qu'il produit aussi bien sur les territoires que pour leurs habitants (Özen et Özen, 2011; Conde, 2017). La convergence des luttes autour des projets du sous-sol pour la transition énergétique se retrouve ainsi cristallisée dans l'opposition à ce modèle de développement capitaliste et extractiviste, dont les critiques mettent tour à tour en avant les enjeux de gouvernance des territoires et ceux de leur préservation biophysique. À travers la critique extractiviste, c'est la relation de la communauté à son environnement qui est mis au cœur du débat et la question qui se pose est celle de l'organisation politique qui permet à des compagnies, souvent étrangères et toujours motivées par les cours des matières premières et de leurs propres actions boursières, de venir exploiter et déstructurer - un territoire au profit quasi exclusif d'acteurs extérieurs qui ne rendent que rarement compte des effets négatifs de leurs activités à moyen et long terme.

Les mouvements sociaux du sous-sol entretiennent également une proximité forte avec les mobilisations contre les "grands projets inutiles et imposés » qui ont gagné en visibilité et en résultats dans la dernière décennie (Les plumes dans le goudron, 2018). La multiplication des ZAD, de Notre-Dame-des-Landes à Roybon en passant par Sivens, la lutte contre la ligne de TGV Lyon-Turin ou encore les actions directes qui se sont tenues à Pau en avril 2016 contre l'extraction du pétrole offshore (à l'occasion d'un sommet organisé par Total) sont les signaux forts d'une telle montée en puissance. Concernant les sous-sols, on note la mobilisation de 2010-2011 contre l'exploitation du gaz de schiste, qui a contribué à en faire échouer l'exploitation en France (Chailleux, 2016), le mouvement contre le projet Cigeo de stockage de déchets nucléaires à Bure ou encore la fronde bretonne contre les projets miniers, abandonnés en 2017. Tous ces mouvements se fondent sur le registre d'une urgence environnementale globale, de risques locaux et de la dénonciation d'un modèle de développement socio-économique des territoires.

Cette problématisation multisituée remet au cœur du débat la possibilité de choisir l'opportunité de telle ou telle utilisation du sous-sol. Malgré le confinement des procédures officielles, les mobilisations sociales autour du sous-sol sont parvenues à questionner de multiples aspects de l'organisation politique et économique des utilisations du sous-sol. La fin du récit moderniste fragilise les justifications passées des projets concernés et les contestations émergentes autour des utilisations du sous-sol mettent en lumière la nécessité d'une refonte fondamentale des structures organisant les utilisations du sous-sol. Mais, malgré cette problématisation croissante $\mathrm{du}$ sous-sol, la traduction en programme d'action politique est encore inachevée.

\section{Une politisation incomplète}

Les contestations contribuent à problématiser le soussol, mais aucune n'a engendré de transformations profondes de la régulation, même si l'interdiction de la fracturation hydraulique en 2011 et l'abandon des permis miniers entre 2017 et 2019 constituent des décisions importantes.

\section{La politisation comme construction d'un enjeu collectif et légitimation de représentations sociales}

Concept central en sciences politiques, la politisation couvre initialement le domaine restreint de la diffusion des clivages politiques dans les sociétés démocratiques (Déloye et Haegel, 2019). Nous 1'employons ici dans une acceptation beaucoup plus large, telle qu'elle s'est développée dans la littérature sur la construction des problèmes publics (Gusfield, 1981; Zittoun, 2013). La politisation décrit d'une part l'interpellation des pouvoirs publics suite à la problématisation d'un enjeu collectif (cadrage, détermination de victimes et de coupables, production d'histoires causales, etc.) et d'autre part le processus de légitimation de (nouvelles) représentations sociales au sein d'un secteur d'activité (Smith, 2019). La politisation est ici envisagée comme l'action de rendre politique un objet (Lagroye, 2003) ou de le mettre en politique (Barthe, 2006), c'est-à-dire de demander l'action d'acteurs politiques pour le réguler et plus largement d'engager un débat politique sur sa valeur, ses représentations et sa signification pour différents collectifs d'individus (D'Arcimoles et Borraz, 2003). 
Dans cette introduction, nous soutenons l'idée d'une politisation nouvelle des sous-sols, qui reste incomplète. La politisation est nouvelle, car de nouveaux enjeux collectifs sont construits et de nouvelles représentations sociales sont diffusées, reposant sur un appel à des valeurs jusque-là peu présentes dans les organisations régulant ses utilisations : leur rôle dans les changements globaux, leur ancrage dans des projets de territoires, leur sens pour les communautés et leurs modes de gouvernance. Depuis la fin des années 2000, un nombre croissant d'acteurs participe ainsi à requalifier le sous-sol comme objet de préoccupations environnementale et politique, et non uniquement autour des enjeux sociaux et sanitaires relatifs aux effets sur la surface des activités d'extraction (Akrich et al., 2010).

Toutefois, cette politisation n'est pas suffisante pour conduire à une politique claire et assumée vis-à-vis du sous-sol. C'est un processus encore largement en cours, soit, selon les cas, que le problème n'a pas atteint l'agenda politique, soit que la solution politique envisagée pour résoudre ce dernier aborde les effets en aval et non pas la source de la problématisation. L'absence d'automaticité entre la mise à l'ordre du jour médiatique d'un problème et la réaction d'un gouvernement a d'ailleurs été à l'origine du concept d' "agenda » lui-même et de la distinction proposée originellement par Cobb et Elder entre systemic agenda et institutionnal agenda, le premier renvoyant essentiellement à l'ordre du jour médiatique et le second à l'ordre du jour gouvernemental (Elder et Cobb, 1984). On observe alors deux tendances. Dans certains cas, comme celui du stockage géologique de $\mathrm{CO}_{2}$, la contestation est canalisée dans des mécanismes consultatifs encadrés conduisant à confiner le problème et in fine à éviter tout enrôlement de personnalités politiques et ainsi tout positionnement public, politique ou stratégique sur le sujet. Dans d'autres cas, comme celui du gaz de schiste ou des permis miniers, la mise en cause directe de l'État conduit bien à la mise à l'agenda politique hors des cadres institutionnels de consultation et à des transformations de politiques sans toutefois toucher au cœur du système; le Code minier, qui organise les procédures, détermine la répartition des richesses et planifie la hiérarchie des activités du sous-sol et de surface. En effet, la mise en politique du sous-sol n'a conduit jusqu'à présent qu'à éviter les problèmes en amont plutôt qu'à traiter les problèmes en aval, c'est-à-dire à travers une interrogation globale sur le rôle des sous-sols dans la transition énergétique, leur mode de gouvernance collective et la répartition des avantages et des inconvénients des utilisations du sous-sol. S'il faut noter un pas dans ce sens à travers la loi Hulot de 2017 sur les hydrocarbures, puisque la loi anticipe la fin de la production d'hydrocarbures en 2040 afin de respecter un objectif de neutralité carbone, force est de constater d'une part l'affaiblissement des ambitions initiales sur les hydrocarbures et d'autre part l'absence de stratégie nouvelle concernant le rôle du sous-sol et le type d'infrastructures nécessaires à une transition juste (Newell et Mulvaney, 2013). L'ensemble des utilisations du sous-sol que nous abordons sont dépendantes du Code minier et, malgré des tentatives de réforme depuis 2011, aucune n'a encore abouti, à cause d'un équilibre des forces au sein même de l'État entre partisans du code productiviste existant et défenseurs du Code de l'environnement.

Il faut comprendre cette politisation incomplète dans sa tension avec un processus de neutralisation des débats par technicisation et naturalisation (Swyngedouw, 2010). En effet, en matière de changements climatiques, Aykut et Dahan (2015) ont montré comment le schisme de réalité était né d'un décalage entre le cadrage scientifique et le cadrage sociopolitique. Dans le cas du sous-sol, un schisme similaire opère entre les promoteurs de solutions techniques imaginées dans les laboratoires et au sein de cercles professionnels et experts, et les mouvements sociaux d'opposants qui en pointent le caractère hors sol et en critiquent les objectifs. La technicisation (Ellul, 1954, 1977, 1988) ou la naturalisation d'un problème public constitue un puissant frein à sa politisation. La forte médiation technique pour la mobilisation du soussol constitue à n'en pas douter un frein à sa politisation.

Il ne faudrait pourtant pas croire que la technicisation offre aux porteurs de projets un chemin sans encombre vers le succès. En évitant de traiter les problèmes en amont, ces derniers sont confrontés à des éruptions sociales de plus en plus fréquentes à mesure que l'urgence environnementale gagne en visibilité. Loin de permettre les projets, cette technicisation est un frein majeur à leur déploiement. Il convient donc de s'interroger sur les conditions nécessaires au développement d'utilisations du sous-sol pour la transition énergétique et de mettre en avant les dimensions techniques et politiques des différentes filières présentes en France durant la dernière décennie.

La notion de technofix permet d'appréhender le phénomène. Ce concept a été élaboré par Alvin $\mathrm{M}$. Weinberg (1967) pour qualifier ces technologies (souvent venues de la géo-ingénierie) qui, supposées résoudre le changement climatique, détournent de politiques structurelles à même de s'attaquer aux racines du problème. Dans un article récent revenant sur cette notion, Daniel Compagnon (2019) montre que les technofixies manquent de trois dimensions essentielles à leur mise en œuvre: la crédibilité de la solution qu'elles sont supposées permettre, la gouvernance du dispositif sociotechnique qui porte la technologie et sa légitimité. Nous reprenons ces trois dimensions afin de caractériser les conditions nécessaires au déploiement d'utilisations du sous-sol pour la transition énergétique. 
La crédibilité est ce qui permet à une technologie de remplir une vraie fonction. Dans son article, Daniel Compagnon insiste sur le fait que les technologies de la géo-ingénierie sont fondées sur de fausses promesses : le stockage géologique du $\mathrm{CO}_{2}$, par exemple, est très loin de pouvoir tenir les objectifs d'émissions négatives de $\mathrm{CO}_{2}$ attendues pour tenir l'augmentation de la température moyenne de l'atmosphère au-dessous des deux degrés (voir aussi McGlade et Ekins, 2015). La crédibilité d'une filière ou d'une technologie est sa capacité à tenir ses promesses à l'échelle locale en matière de gestion des risques, à l'échelle nationale en matière d'intégration dans un marché et à l'échelle globale en matière de lutte contre le réchauffement climatique.

La gouvernance d'une technologie repose sur la configuration institutionnelle encadrant l'activité qui permet à un nombre plus ou moins large d'acteurs d'intervenir dans le processus décisionnel, comme les permis, mais aussi dans la régulation de l'activité et l'évaluation des risques. Certes, toute technologie est toujours présentée comme devant être bien portée pour être mise en œuvre. C'est notamment le cœur des principes d'Oxford supposés permettre un bon déploiement de la géo-ingénierie (Shepherd et al., 2009; Rayner et al., 2013). Mais la gouvernabilité d'une technologie va plus loin qu'une bonne gouvernance du déploiement de la technologie: elle suppose que la recherche menée en amont de la technologie soit faite de manière consciente et discutée, que le développement de la technologie ne soit pas encapsulé dans des dispositifs qui ne permettent pas sa modification, que sa territorialisation permette un refus et/ou une modification de la technologie pour qu'elle s'adapte à son environnement.

Enfin, la légitimité vient décrire le consentement plus ou moins grand de différentes catégories d'acteurs au projet. La légitimité s'appréhende au regard des principes démocratiques qui gouvernent le déploiement d'une technologie, mais aussi des objectifs qu'elle est supposée remplir - la lutte contre un problème social. La légitimité est construite sur une relation de confiance envers les acteurs qui portent un projet et les caractéristiques et objectifs du projet lui-même. La légitimité est à la fois liée aux acteurs et aux résultats qu'ils promettent, mais aussi au processus par lequel le projet advient (Schmidt, 2013).

Ces trois conditions sont nécessaires pour qu'une technologie passe du stade de simple proposition, voire de technofix, à celui de politique crédible pour permettre au sous-sol de remplir la fonction qui est la sienne dans la lutte contre le changement climatique. Or c'est bien ce qui semble manquer aux utilisations du sous-sol étudiées dans ce numéro spécial.

\section{Une diversité d'utilisations du sous-sol qui souligne son incomplète politisation}

Mis en regard de cette grille de la politisation des technologies, les articles réunis dans ce numéro spécial montrent que les mobilisations du sous-sol ne semblent qu'imparfaitement parvenir à remettre le sous-sol dans les politiques contemporaines en leur conférant une utilité sociale ou économique crédible.

Les articles traitent de toutes les technologies participant de la politisation du sous-sol pour la transition énergétique. Deux d'entre eux portent sur des usages extractifs du sous-sol : l'un sur le renouveau minier (Brice Laurent et Julien Merlin), l'autre sur des usages d'énergies fossiles supposées être moins émettrices de $\mathrm{CO}_{2}$ que le pétrole et le charbon (bridge fuels), comme le gaz de houille ou de couche (Olivier Labussière). Trois autres articles portent sur des usages qui permettent de faire du stockage géologique, l'un de $\mathrm{CO}_{2}$ (Sébastien Chailleux et Xavier Arnauld de Sartre), l'autre de déchets radioactifs (Julie Blanck) et le troisième de déchets (Thomas Schellenberger). Enfin, le dernier article porte sur la seule technologie qui se propose de produire des énergies décarbonées à partir du sous-sol: la géothermie (Philippe Chavot, Anne Masseran, Yeny Serrano et Jean Zoungrana). À l'exception du stockage d'hydrogène vert ou de la production d'hydrogène natif, qui sont des technologies loin d'être arrivées à maturité, toutes les formes de mobilisation des sous-sols pour la transition énergétique sont traitées dans ce numéro spécial.

Chacune de ces formes aborde une ou plusieurs thématiques qui permettent d'appréhender la question de la politisation du sous-sol. La question de la crédibilité est posée par l'article de Brice Laurent et Julien Merlin, qui interroge le rôle particulier joué par les compagnies juniors dans l'exagération du potentiel économique du renouveau minier - et donc dans une dimension de sa mise en politique. Les auteurs pointent la difficulté des acteurs industriels à rester crédibles sur plusieurs tableaux à la fois entre le public des actionnaires et celui des riverains. L'article que nous avons écrit traite, lui, du fait que les défauts de crédibilité, à la fois en termes d'efficacité et de marché économiques, ont empêché qu'un véritable débat se développe autour du stockage géologique de $\mathrm{CO}_{2}$ : non crédible, le stockage géologique de $\mathrm{CO}_{2}$ ne fait pas l'objet d'une politisation organisée et structurée. Là encore, l'article souligne que la crédibilité du stockage de $\mathrm{CO}_{2}$ se joue sur trois tableaux, celui de la crédibilité technique, économique et au regard des changements climatiques - et que rien n'est acquis dans cette argumentation.

Deux articles traitent des manières de gouverner le déploiement des technologies: celui de Thomas 
Schellenberger aborde la question des droits d'accès au sous-sol pour le stockage de déchets et de $\mathrm{CO}_{2}$. Ces deux types de résidus industriels sont envisagés de concert afin de souligner leurs points communs et leurs différences sous l'angle juridique. Cet article analyse les règles d'appropriation des espaces souterrains aptes aux stockages géologiques. Il en ressort que la manière dont le droit encadre la gestion de ces externalités de la modernité fait du sous-sol un espace à disposition des industriels pour faciliter leurs activités et la gestion de leurs externalités (déchets et $\mathrm{CO}_{2}$ ). De son côté, Olivier Labussière montre comment une compagnie d'exploration parvient à enrôler les héritages locaux dans la promotion d'une nouvelle source de gaz «lorrain». En développant l'analyse de l'identification d'une ressource et de caractérisation, l'auteur retrace les processus sociotechniques contribuant à construire une ressource pour la transition énergétique.

La question de la légitimité est abordée dans tous ces articles, mais elle est reprise très directement dans deux d'entre eux: celui de Julie Blanck, qui parle de la construction de la légitimité pour le stockage des déchets radioactifs, mais en montrant que cette légitimité est très largement inachevée dans la mesure où les structures de gouvernance de la construction de la légitimité excluent les opposants les plus radicaux, et celui de Philippe Chavot et ses coauteurs, qui montre comment la légitimité est inégalement acquise dans le débat public selon l'ancrage territorial des porteurs de technologies.

Bien entendu, d'autres lectures de ces articles sont possibles. Mais il nous apparait qu'un de leurs points communs est qu'ils montrent tous, à leur manière, comment le développement, de manière non coordonnée, de nouvelles utilisations du sous-sol se heurte à de nombreuses difficultés qui, du moins est-ce notre propos, trouvent leur origine dans l'incomplète politisation du sous-sol. L'entretien mené avec Carole Mercier, qui a travaillé dans différents services de l'administration centrale française en charge de l'exploitation ou de la gestion du sous-sol, confirme cette vision, du point de vue cette fois d'un agent des bureaux en charge - justement - de coordonner cette politique. L'entretien montre notamment comment le soussol est progressivement sorti du champ politique pour devenir une affaire technique, administrative, ce qui l'a éloigné des débats de son temps et n'a pas permis de portage politique des enjeux liés à ce milieu... ce qui s'est révélé préjudiciable à l'exploitation des ressources de ce milieu quand les contestations ont émergé. Les différents comptes rendus complètent ce panorama des utilisations du sous-sol en discutant les ouvrages récents traitant du sous-sol et de ses contestations.

\section{Conclusion}

Mobilisations en faveur ou en défaveur du sous-sol ne valent pas, tant s'en faut, une mise en débat et ne permettent pas la construction d'un choix politique. Si les mobilisations permettent un enrôlement politique autour d'un objet au nom d'une lutte, la construction politique du sous-sol comme objet d'un programme politique implique une régulation du milieu qui suit une stratégie claire, assumée, soumise au débat démocratique - ce qui apparaît comme la condition à la mise en œuvre des projets. Nous estimons que le processus de politisation du sous-sol est incomplet du fait que certaines dimensions des projets ne sont pas discutées publiquement et n'aboutissent pas à des choix politiques clairs, soit que la crédibilité est incertaine, soit que la gouvernance est inégale, soit que la légitimité est contestée.

Cela s'explique bien évidemment d'abord par l'ambivalence du sous-sol au regard de la transition énergétique: les matières contenues dans le sous-sol permettent en effet tout à la fois de générer et d'atténuer les changements climatiques. Cette ambivalence ne saurait cependant à elle seule expliquer le défaut de politisation et donc de choix: les particularités de ce milieu, qui requiert une médiation technique pour être connu et exploité, ne jouent pas en faveur d'une mise en débat. La médiation technique est en effet très souvent un puissant facteur de dépolitisation des problèmes - d'autant que l'importance des capitaux nécessaires pour exploiter ce milieu l'associe souvent à un capitalisme concentré. Confinées dans des arènes techniques dont sont exclues les mobilisations citoyennes, les technologies du sous-sol se prêtent mal sinon au débat, du moins à l'arbitrage des débats. D'où sans doute les formes d'actions très éruptives auxquelles elles donnent lieu.

\section{Références}

Akrich M., Barthe Y., Rémy C., 2010. Sur la piste environnementale. Menaces sanitaires et mobilisations profanes, Paris, Presses des Mines.

Aykut, S., Castro-Larrañaga, M., 2017. The end of fossil fuels? Understanding the partial climatisation of energy policy, in Aykut, S., Foyer, J. and Morena, E. (Ed.), Globalising the climate: COP 21 and the climatisation of global debates, London, Routledge, 173-193.

Aykut S.C., Dahan A., 2015. Gouverner le climat? 20 ans de négociations internationales, Paris, Presses de Sciences Po.

Barthe Y., 2006. Le pouvoir d'indécision la mise en politique des déchets nucléaires, Paris, Economica. 
Batellier P., 2016. Acceptabilité sociale des grands projets à fort impact socio-environnemental au Québec: définitions et postulats, VertigO - la revue électronique en sciences de l'environnement, 16, 1, [En ligne] mis en ligne le 9 mai 2016, consulté le 20 février 2017, http://vertigo.revues.org/ 16920.

Bebbington A., Bury J. (Eds.), 2013. Subterranean Struggles: New Dynamics of Mining, Oil and Gas in Latin America, Austin, University of Texas Press.

Bednick A., 2015. Extractivisme - Exploitation industrielle de la nature: logiques, conséquences, résistances, Paris, Le passager clandestin.

Boissonade J., Barbier R., Bauler T., Fortin M.-J., Fournis Y., Lemarchand F., Raufflet E., 2016. Mettre à l'épreuve l'acceptabilité sociale, VertigO - la revue électronique en sciences de l'environnement, 16, 1, [En ligne] mis en ligne le 9 mai 2016, consulté le 20 février 2017, http://vertigo. revues.org/17163.

Bonneuil C., Fressoz J.-B., 2013. L'Événement Anthropocène. La Terre, l'histoire et nous, Paris, Le Seuil, coll. « Anthropocène ».

Braun B., 2000. Producing vertical territory: geology and governmentality in late Victorian Canada, Ecumene, 7, 1, 746, https://www.jstor.org/stable/44252276.

Chailleux S., 2016. Incertitude et action publique. Définition des risques, production des savoirs et cadrage des controverses, Revue internationale de politique comparée, 23, 4, 519-548, https://doi.org/10.3917/ripc.234.0519.

Chailleux S., 2020. Making the subsurface political: How enhanced oil recovery techniques reshaped the energy transition, Environment and Planning C, 38, 4, 733-750, https://doi.org/10.1177\%2F2399654419884077.

Chailleux S., Merlin J., Gunzburger Y., 2018. Unconventional oil and gas in France: From popular distrust to politicisation of the underground, The extractive industries and society, 5, 4, 682-690, https://doi.org/10.1016/j.exis.2018.05.007.

Clark N., 2016. Politics of Strata, Theory, Culture \& Society, 34, 2-3, 211-231, https://doi.org/10.1177/ 0263276416667538 .

Compagnon D., 2019. Governing a Mirage? False Promises of Negative Emissions Technologies, Carbon \& Climate Law Review, 13, 2, 104-112, https://halshs.archives-ouvertes.fr/ halshs-02279735.

Conde M., 2017. Resistance to mining. A review, Ecological Economics, 132, 80-90, https://doi.org/10.1016/j.ecole con.2016.08.025.

D’Arcimoles M., Borraz O., 2003. Réguler ou qualifier. Le cas des boues d'épuration urbaine, Sociologie du Travail, 45, 45-62, https://doi.org/10.4000/sdt.30866.

Déloye Y., Haegel F., 2019. La politisation: du mot à l'écheveau conceptuel, Politix, Louvain-la-Neuve, De Boeck Supérieur, 127, 3, 59-83, https://hal.archivesouvertes.fr/hal-02410138/.

Elder C.D., Cobb R.W., 1984. Agenda building and the politics of aging, Policy Studies Journal, 13, 1, 115-129, https://doi. org/10.1111/j.1541-0072.1984.tb01704.x.
Ellul J., 1954. La Technique, ou l'enjeu du siècle, Paris, Economica.

Ellul J., 1977. Le Système technicien, Paris, Le Cherche midi.

Ellul J., 1988. Le bluff technologique, Paris, Hachette, Pluriel (2012).

Fressoz J.-B., 2012. L'apocalypse joyeuse. Une histoire du risque technologique, Paris, Le Seuil.

Gourgues G., 2013. Les politiques de démocratie participatives, Grenoble, Presses Universitaires de Grenoble.

Gourgues G., Rui S., Topçu S., 2013. Gouvernementalité et participation. Lectures critiques, Participations, 62, 5, https://doi.org/10.3917/parti.006.0005.

Gudynas E., 2009. Diez tesis urgentes sobre el nuevo extractivismo: contextos y demandas bajo el progresismo sudamericano actual, in Schludt J., Acosta A., Barandiarán A., Bebbington A., Flochi A., Alayza A., Gudynas E. (Eds.), Extractivismo, politica y sociedad, Quito, CAAP/ Claes, 187-225.

Gusfield J., 1981. The Culture of Public Problems. Drinking, Driving and the Symbolic Order, Chicago, University of Chicago Press.

Huber M.T., 2009. Energizing historical materialism: Fossil fuels, space and the capitalist mode of production, Geoforum, 40, 1, 105-115, https://doi.org/10.1016/j.geofo rum.2008.08.004.

Kirat T., Torre A. (Eds.), 2008. Territoires de conflits Analyses des mutations de l'occupation de l'espace, Paris, L'Harmattan.

Krause R.M., Carley S.R., Warren D.C., Rupp J.A., Graham J. D., 2014. 'Not in (or under) my backyard': Geographic proximity and public acceptance of carbon capture and storage facilities, Risk analysis: an official publication of the Society for Risk Analysis, 34, 3, 529-540, https://doi.org/ 10.1111/risa.12119.

Lagroye J., 2003. La politisation, Paris, Belin.

Les plumes dans le goudron, 2018. Résister aux grands projets inutiles et imposés, Paris, Textuel.

McGlade C., Ekins P., 2015. The geographical distribution of fossil fuels unused when limiting global warming to 2 [deg] C, Nature, 517, 7533, 187-190, https://doi.org/10.1038/ nature 14016.

Mitchell T., 2013. Carbon democraty. Le pouvoir politique à l'ère du pétrole, Paris, La Découverte.

Moore J.W., 2017. The Capitalocene, Part I: on the nature and origins of our ecological crisis, The Journal of Peasant Studies, 44, 3, 594-630, https://doi.org/10.1080/ 03066150.2016 .1235036 .

Mumford L., 2016. Technique et civilisation, Paris, Parenthèses.

Newell P., Mulvaney D., 2013. The political economy of the 'just transition': The political economy of the 'just transition', The Geographical Journal, 179, 2, 132-140, https://doi.org/10.1111/geoj.12008.

Özen H., Özen S., 2011. Interactions in and Between Strategic Action Fields: A Comparative Analysis of Two Environmental Conflicts in Gold-Mining Fields in Turkey, 
Organization Environment, 24, 3, https://doi.org/10.1177/ 1086026611426343.

Pessis C., Topçu S., Bonneuil C., 2013. Une autre histoire des "Trente Glorieuses ». Modernisation, contestations et pollutions dans la France d'après-guerre, Paris, La Découverte, coll. « Cahiers libres ».

Pomeranz K., 2010. Une grande divergence. La Chine, l'Europe et la construction de l'économie mondiale, Paris, Albin Michel, Éditions de la Maison des Sciences de l'Homme.

Rayner S., Heyward C., Kruger T., Pidgeon N., Redgwell C., Savulescu J., 2013. The Oxford Principles, Climatic Change, 121, 3, 499-512, https://doi.org/10.1007/s10584-012-0675-2.

Schmidt V.A., 2013. Democracy and Legitimacy in the European Union Revisited: Input, Output and 'Throughput', Political Studies, 61, 1, 2-22, https://doi.org/10.1111/ j.1467-9248.2012.00962.x.

Shepherd J., Cox P., Haigh J., Keith D., Launder B., Mace G., Mackerron G., Pyle J., Rayner S., Redgwell C., Watson A., 2009. Geoengineering the climate: science, governance and uncertainty, London, The Royal Society.
Smith A., 2019. Travail politique et changement institutionnel: une grille d'analyse, Sociologie du travail, 61, 1, https://doi. org/10.4000/sdt.14661.

Svampa M., 2016. Reconfiguration du clivage Nord-Sud et géographie de l'extraction, Alternatives Sud, 23.

Swyngedouw E., 2010. Apocalypse Forever?, Theory, Culture \& Society, 27, 2-3, 213-232, https://doi.org/10.1177/ 0263276409358728.

Trom D., 1999. De la réfutation de l'effet NIMBY considérée comme une pratique militante. Notes pour une approche pragmatique de l'activité revendicative, Revue Française de Science Politique, 49, 1, 31-50.

Weinberg A.M., 1967. Can technology replace social engineering?, Bulletin of the Atomic Scientists, 10, 9, 4-8, https://doi.org/10.1080/00963402.1966.11454993.

Wylie S.A., 2018. Fractivism: corporate bodies and chemical bonds, Durham, Duke University Press, coll. Experimental futures.

Zittoun P., 2013. La fabrique politique des politiques publiques. Une approche pragmatique de l'action publique, Paris, Presses de Sciences Po. 